\title{
Revamped Rationing of Renal Resources: Kidney Allocation in Search of Utility and Justice for All
}

\author{
Steven C. Kim ${ }^{1} \cdot$ Thomas C. Pearson ${ }^{2} \cdot$ Paul L. Tso ${ }^{2}$
}

Published online: 28 March 2015

(C) Springer International Publishing AG 2015

\begin{abstract}
The widening gap between patients in need of a kidney transplant and the finite number of transplantable deceased donor organs resulted in critical review of kidney allocation policy in the USA. The process to reach consensus for change required compromise between arguments for utility and equity. Important amendments to address shortcomings of the old system and projected outcomes of the new system are reviewed. Potential unintended consequences and practical considerations to facilitate implementation of provisions intended to enhance utility and increase access to transplant for population subgroups in the new system are explored. Granular metrics provide built-in flexibility for the new system, making it amenable to modification based on outcomes.
\end{abstract}

Keywords Kidney $\cdot$ Transplant $\cdot$ Allocation $\cdot$ Rationing · $\mathrm{KTC} \cdot \mathrm{OPTN}$

This article is part of the Topical Collection on Kidney Transplantation

Steven C. Kim

sckim@emory.edu

Thomas C. Pearson

tpearso@emory.edu

Paul L. Tso

ptso@emory.edu

1 Department of Surgery, Emory University Hospital, Room B206, Atlanta, GA 30322, USA

2 Emory University Transplantation Immunology, Woodruff Memorial Research Building 101 Woodruff Circle, Atlanta, GA 30322, USA

\section{Introduction}

The widening gap between the increasing number of candidates waiting for a kidney and the finite supply of transplantable deceased donor organs over the past few decades brought forth calls to critically review kidney allocation policy in the USA. The push to overhaul the renal allocation system officially began over a decade ago in 2003 to address limitations of the status quo. In light of the aging population and propensity for end-stage renal disease to affect the elderly, one prominent concern is that many kidneys with long projected posttransplant graft survival may be allocated to candidates with relatively short post-transplant life expectancy under the current or "old" system based primarily on waiting time. Conversely, candidates with the greatest life expectancy and, therefore, best served with long-functioning allografts may be offered kidneys with relatively shorter expected graft survival. This situation results in excessive unrealized potential graft years, increased numbers of patients with failed allografts to be added to the wait list again and unnecessarily high retransplant rates. Also, the number of transplant opportunities for certain disadvantaged groups, for instance patients with biological factors such as blood type B or high level of donor-specific antibody (DSA) may be disproportionately low under the old rules. Another major issue of concern is inefficient placement of organs at higher risk of graft failure, specifically, expanded criteria donor (ECD) kidneys, resulting in higher than necessary discard rates of transplantable organs.

The process to reach consensus for changes to kidney allocation generated much needed debate which has been heated, incompletely resolved, and frequently divided along the polarizing principles of utility vs. justice as well documented in the scientific as well as lay literature. However, the tireless 
efforts of the Kidney Transplantation Committee (KTC) of the Organ Procurement and Transplantation Network (OPTN) are coming to fruition as substantial amendments to allocation policy were approved in June 2013 and are anticipated to be implemented in late $2014[1 \bullet \bullet, 2 \bullet \bullet$ (Table 1). For detailed information on new policy development including previous proposals, public debate on points of controversy, accepted amended allocation policy, and KTC reports, the interested reader is referred to the OPTN website at http://optn. transplant.hrsa.gov/. Important changes intended to address deficiencies of the old system (Table 2) and projected outcomes of the new system are herein reviewed. Potential unintended sequelae and practical considerations to facilitate implementation of provisions intended to enhance utility and increase access to transplant for population subgroups in the new system are explored (Table 3).

\section{Key Concepts of the New Allocation System}

The new system will allocate kidneys based upon projected longevity of the organ as quantified by the kidney donor profile index (KDPI), a relatively new metric developed to assess organ quality. Derived from the kidney donor risk index previously described by Rao et al. [3], the KDPI incorporates multiple parameters to give a more granular estimate for risk of graft failure compared to the $\mathrm{ECD} /$ standard criteria donor (SCD) dichotomy used in the old system. Similarly, an estimated post-transplant survival (EPTS) score for transplant candidates will be calculated based on four parameters (age, diabetes status, time on dialysis, and previous transplant status) to enable stratification of candidates awaiting transplant. As the predictive accuracy of the EPTS calculation is recognized to be limited, it will be used only to broadly categorize
Table 1 Timeline for the development of the new kidney allocation policy

\begin{tabular}{|c|c|}
\hline Date & Sentinel event \\
\hline 2003 & $\begin{array}{l}\text { The Kidney Allocation Review Subcommittee (KARS) is instructed by the } \\
\text { Organ Procurement and Transplantation Network (OPTN) Board of } \\
\text { Directors to conduct a } 360^{\circ} \text { review of the current (old) allocation system. } \\
\text { This process included public hearings to better understand limitations of } \\
\text { the current system and potential approaches for improvement. }\end{array}$ \\
\hline 2004 & $\begin{array}{l}\text { OPTN Board of Directors instructs KARS to explore the use of net lifetime } \\
\text { survival benefit in a new allocation system. }\end{array}$ \\
\hline 2005 & $\begin{array}{l}\text { KARS merges with the OPTN Kidney Transplantation Committee (KTC) } \\
\text { and the formal new policy development process commences. }\end{array}$ \\
\hline 2007 & $\begin{array}{l}\text { Public forum held in Dallas, TX reviews the use of life years from } \\
\text { transplant (LYFT) in an allocation system. }\end{array}$ \\
\hline September 2008 & $\begin{array}{l}\text { Request for information (RFI) issued detailing new concepts of LYFT, } \\
\text { kidney donor profile index (KDPI), and changes to wait time calculation } \\
\text { to include time on dialysis prior to listing. }\end{array}$ \\
\hline January 2009 & $\begin{array}{l}\text { Public forum held in St. Louis, MO to review concepts recently circulated } \\
\text { in September 2008. Participants included representatives from the } \\
\text { American Association of Kidney Patients, American Society of } \\
\text { Histocompatibility and Immunogenetics, American Society of Transplant } \\
\text { Surgeons, American Society of Transplantation, National Association of } \\
\text { Transplant Coordinators, National Kidney Foundation, and Renal } \\
\text { Support Network. }\end{array}$ \\
\hline 2009 & $\begin{array}{l}\text { The KTC considers age matching as a way to address concerns regarding } \\
\text { system complexity at the recommendation of forum participants. }\end{array}$ \\
\hline February 2011 & $\begin{array}{l}\text { Concept document released detailing the use of estimated post-transplant } \\
\text { survival (EPTS), age matching } \pm 15 \text { years of donor and recipient, } \\
\text { and KDPI. }\end{array}$ \\
\hline 2011-2012 & $\begin{array}{l}\text { Committee considers alternatives to age matching after receiving feedback } \\
\text { suggesting that age matching does not meet the requirements of the } \\
1979 \text { Age Discrimination Act. }\end{array}$ \\
\hline September 2012 & $\begin{array}{l}\text { The KTC issues a new proposal incorporating EPTS, KDPI, and changes } \\
\text { to wait time calculation for public comment. }\end{array}$ \\
\hline June 2013 & $\begin{array}{l}\text { After discussion and modification of the new proposal by the KTC based } \\
\text { on public feedback, OPTN Board of Directors approves substantial } \\
\text { amendments to deceased donor kidney allocation policy. }\end{array}$ \\
\hline 2014 & Anticipated implementation of new allocation policy. \\
\hline
\end{tabular}

Based on Table 1 in "Proposal to Substantially Revise the National Kidney Allocation System" issued September 2012, http://optn.transplant.hrsa.gov/PublicComment/pubcommentPropSub_311.pdf, accessed October 13, 2014 
Table 2 Important changes to address deficiencies of old kidney allocation system and increase utility and equity

\begin{tabular}{lc}
\hline Limitations of old kidney allocation system & Revisions to address limitations \\
\hline $\begin{array}{l}\text { Poor longevity matching of recipient and organ } \\
\text { esulting in unrealized graft years and unnecessarily } \\
\text { high re-transplant rates }\end{array}$ & $\begin{array}{c}\text { Longevity matching for a portion of kidneys and } \\
\text { recipients - top 20\% KDPI prioritized to top }\end{array}$ \\
$\begin{array}{l}\text { Higher than necessary rate of discard of ECD kidneys } \\
\text { that could benefit candidates on the waiting list }\end{array}$ & $\begin{array}{c}\text { Regional sharing of kidneys at highest risk of } \\
\text { discard, i.e., KDPI }>85 \% \text { (enhance utility) }\end{array}$ \\
Limited access to transplant for certain subgroups: & \\
Highly sensitized & Sliding scale to assign points for sensitized patients \\
& and broader sharing for extremely highly \\
& sensitized patients (enhance equity) \\
Blood group B & Expanded access for blood type B candidates who \\
& can accept kidneys from blood type A2 and \\
A2B donors (enhance equity) \\
Socially underprivileged & Waiting time redefined to include dialysis time \\
& prior to listing (enhance equity) \\
\hline
\end{tabular}

patients into two groups: the top $20 \%$ longevity group and the lower $80 \%$ longevity group, as detailed in the September 2012 KTC proposal.

The $20 \%$ of kidneys with the longest graft survival as predicted by KDPI will be allocated preferentially to the $20 \%$ of patients with the longest EPTS in the new allocation system (Table 2). This longevity matching is intended to increase realized graft years and decrease the need for a repeat transplant in patients who have relatively longer expected survival. This may in turn curb growth of the wait list and, thus, facilitate other patients to receive a transplant.

In the new system, pediatric candidates in general will still maintain priority over adult candidates for kidney allocation similar to the previous scenario. However, going forward this prioritization will be for organs from donors with KDPI $<35 \%$ rather than donors less than 35 years of age.
The ECD designation will be redefined as all donors with KDPI $>85 \%$ in the new system. Analogous to the ECD categorization, these organs assessed to be at higher risk for graft failure will likely provide survival benefit compared to dialysis and are a reasonable consideration for candidates such as elderly patients for whom the additional survival benefit associated with a lower risk organ is negated by the extra wait time required for such an organ. Candidates will give informed consent to be considered for organs with KDPI $>85 \%$, similar to the requirement for ECD kidneys. In addition, candidates for these organs will be rank-ordered solely by wait time to facilitate wait list management. Kidneys with KDPI $>85 \%$ will initially be offered at the regional level to expedite placement and hopefully increase recovery and utilization of these organs (Table 2).

Waiting time calculation will change under the new system such that it will begin to accrue either from the time of listing with dialysis initiation or with estimated glomerular filtration
Table 3 Concerns arising from new kidney allocation policy and practical measures to facilitate desired outcomes

\begin{tabular}{|c|c|}
\hline Concerns related to new allocation policy & Practical responses to effect desired results \\
\hline $\begin{array}{l}\text { Patients prioritized in top } 20 \% \text { EPTS or with much } \\
\text { pre-listing dialysis time may forgo living donor } \\
\text { transplant opportunities }\end{array}$ & $\begin{array}{l}\text { Encourage living donor transplant as wait time will } \\
\text { still be substantial for prioritized patients and } \\
\text { outcomes are superior with living donor transplants }\end{array}$ \\
\hline $\begin{array}{l}\text { Patients may be less inclined to seek pre-emptive or } \\
\text { early listing for transplant due to crediting wait } \\
\text { time to initiation of dialysis }\end{array}$ & $\begin{array}{l}\text { Encourage early/pre-emptive listing as opportunities } \\
\text { for early transplant will still exist via zero mismatch } \\
\text { and high CPRA offers. Prolonged dialysis correlates } \\
\text { with diminished outcome }\end{array}$ \\
\hline \multirow[t]{2}{*}{ Access to transplant for the elderly may be diminished } & $\begin{array}{l}\text { Increase application of strategies to improve utilization } \\
\text { and outcomes of higher KDPI kidneys, such as } \\
\text { machine perfusion, dual kidney transplants, and } \\
\text { appropriate listing of candidates }\end{array}$ \\
\hline & $\begin{array}{l}\text { Encourage living donor transplant as outcomes are } \\
\text { superior and may ameliorate negative impact of } \\
\text { comorbidity }\end{array}$ \\
\hline
\end{tabular}


rate or creatinine clearance less than $20 \mathrm{ml} / \mathrm{min}$ or from the date dialysis was initiated if dialysis was started before listing. United Network for Organ Sharing (UNOS) data presented at the March 2013 KTC meeting demonstrated that the length of pre-registration dialysis time varies substantially by subgroup, with certain minorities such as African-Americans and Hispanics experiencing longer periods of dialysis prior to listing. These findings are corroborated by multiple studies documenting the correlation between specific demographic or socioeconomic characteristics such as African-American race or lower level of education or income and time to listing for kidney transplantation [4-8]. Crediting wait time points to include all pre-registration dialysis time may provide a more equitable system for these disadvantaged populations (Table 2).

In the new system, additional points will be awarded to sensitized patients with calculated panel reactive antibody (CPRA) $\geq 20 \%$ on a continuous sliding scale which is more reflective of their biology and likelihood of finding an immunologically compatible donor organ. The CPRA is based upon unacceptable HLA antigens as determined by the transplant program based on risk attributed to circulating antibodies in a candidate's serum against those specific HLA antigens. These unacceptable antigens represent a positive virtual crossmatch. CPRA provides an estimate of percentage of deceased organ donors that will be crossmatch incompatible for a candidate $[9,10 \bullet$. There will be prioritization of patients with CPRA $\geq 98 \%$ with regional sharing of kidneys for candidates with CPRA $99 \%$ and national sharing of kidneys for candidates with CPRA $100 \%$. Modeling suggests this approach will improve access to transplant for these patients to be more in line with those who do not face the barriers of sensitization (Table 2).

Kidneys from donors with blood group A2 and A2B will be allocated to suitable blood group B recipients in order to increase transplant rates for patients of this blood type who typically have inordinately long wait times (Table 2). The explanation for this disparity in wait time is multifactorial. Allocation by blood group compatibility is partly responsible: the donor population has been reported to be predominantly comprised of Caucasians, who carry an incidence of blood group B of approximately $9 \%$; whereas, blood type B candidates comprise almost $17 \%$ of the kidney wait list. Almost two thirds of the blood group B list is comprised of minorities; blacks and Asians carry relatively higher frequencies of blood group B (approximately 19 and $25 \%$, respectively) [11]. UNOS previously implemented a voluntary national kidney allocation variance in 2001 to permit organ procurement organizations (OPO's) to preferentially allocate $\mathrm{A} 2$ and $\mathrm{A} 2 \mathrm{~B}$ kidneys to $\mathrm{B}$ candidates to help address this disparity in access $[12 \cdot, 13]$. Under the new policy, transplant centers will be required to develop written policies specifying anti-A titer thresholds for blood group B candidates to be eligible for transplant and confirm/update candidate eligibility every
90 days. Blood group B patients willing to accept these kidneys will be required to give consent to be transplanted under this provision of the new policy.

\section{Projected Outcomes of New Allocation Policy}

The distribution of transplants and outcomes predicted by simulation modeling under the new allocation system compared to old rules are not surprising. The kidney-pancreas simulated allocation model (KPSAM) program used by the OPTN projects more transplants for adults under 50 and fewer transplants for candidates 50 and over under the new system. It also predicts more transplants for blood group B candidates and fewer for blood group A patients. The number of transplants in sensitized patients with CPRA $>20 \%$ is also expected to increase. Regional sharing of kidneys for recipients with CPRA $99 \%$, national sharing for candidates with CPRA $100 \%$, and regional sharing of kidneys with KDPI $>85 \%$ are expected to bring about a slight overall increase in kidneys shared beyond the local area $[1 \bullet \bullet$.

The approved amendments to kidney allocation are expected to improve median patient and graft survival compared to the old system (12.65 vs. 11.82 and 9.07 vs. 8.82 years, respectively) [1••]. A model presented at the March 2013 KTC meeting projected that the new allocation system would result in cost savings estimated at $\$ 230,000,000$ for the first year and $\$ 47,000,000$ in the following years. These outcomes are largely attributable to the increase in earlier transplants for patients in the top $20 \%$ EPTS and the associated decrease in morbidity, mortality, and cost associated with long-term dialysis. It is notable that predicted improvement in survival metrics and likely cost savings associated with the approved amendments to allocation policy are somewhat less than projected with earlier proposals which had greater emphasis on longevity or survival matching. This reflects the dynamic tension between justice- and utility-driven arguments and the need for compromise encountered in the process of developing a new allocation system. Ultimately, it was felt reasonable to accept the trade-off of decreased gains in utility measures in order to increase access to transplant for disadvantaged subgroups such as highly sensitized patients and to maintain relative equity across age groups in the new system.

\section{Limitations of Modeling and Potential Unintended Sequelae of New Policy}

Although organ allocation policy is heavily influenced by modeling used to forecast the results of potential changes to the system, the projections generated through simulation may not be entirely reliable. As pointed out by Schold and Reese [14•], the KPSAM-derived estimates for 2010 under the old 
system were not entirely concordant with actual observed outcomes for the same calendar year. Many of the limitations of modeling are related to the unpredictability of human behavior such as changes in organ acceptance patterns under a new allocation system which may be difficult or impossible to account for in simulation. The greatest gains in survival metrics are expected to be derived from longevity pairing of candidates in the top $20 \%$ EPTS with kidneys in the top $20 \%$ KDPI. A potential pitfall is that this group of recipients, comprised largely of young adults, may be at higher risk for nonadherence to medications or other aspects of the posttransplant regimen [15], theoretically compromising projected gains in graft- and life-years under the new system $[1 \bullet \bullet$. There has also been demonstrated complexity of donor and recipient variables and their interactions $[16,17]$ which are likely not fully encompassed in a computer program. KPSAM modeling predicted outcomes based in part upon a rise in the number of patients with prolonged dialysis time projected to undergo transplant under provisions of the new system. A subtle but relevant observation is that dialysis time prior to listing correlates more strongly with poor outcome post-transplant than dialysis time accrued after listing [4]. The new policy will conceivably transplant more patients who have longer prelisting dialysis exposure; as a result, actual gains in survival may be less than projected in simulation [14•]. Likewise, if increased risks for rejection and diminished graft survival in highly sensitized patients are not accurately accounted for, there may be disparity between projected and actual outcomes under the new system prioritizing high CPRA patients for transplant $[1 \cdot \bullet]$.

Another concern is that the patients in the top $20 \%$ EPTS may be more likely to forgo living donor transplant opportunities due to the impression that they are more likely to receive a high-quality deceased donor organ with little or no waiting time under the new allocation policy. Conceivably, other candidates who have accumulated much dialysis time prior to listing also may now be less inclined to seek a living donor due to the perception that the new system places them higher on the waiting list (Table 3). Observers point to the decline in number of living donor renal transplants performed in children after pediatric recipients were given priority for all donors under 35 years of age, a trend which has been attributed at least in part to the potential opportunity to receive an ideal deceased donor organ with little or no wait time [2••].

Accompanying the change in waiting time calculation is the potential significant shift in rank-ordering of candidates on the list and associated issues with list management. A more specific concern is that patients may be less inclined to seek pre-emptive or early listing for transplant in the new system as they may believe that their opportunity for an early deceased donor transplant is diminished in light of so many other patients with long-standing ESRD who will have their wait-time back-dated to the initiation of dialysis (Table 3).

\section{Practical Considerations to Effect Desired Changes in the New System}

In contrast to the pediatric wait list scenario, it appears that in all donation service areas, the number of kidneys fulfilling the top $20 \%$ of KDPI is greatly eclipsed by the number of adult candidates with EPTS in the top $20 \%$. Also, within each allocation sequence ranking of candidates will still be determined by several factors used in the old system, notably qualified wait time, degree of HLA-DR matching and level of sensitization. Therefore, although time to transplant for adult candidates in the top $20 \%$ EPTS is anticipated to be shorter than for the remaining $80 \%$ under the new policy, it is still projected to be substantial $[1 \bullet \cdot 2 \bullet \cdot]$. The transplant community should therefore continue to emphasize to these relatively advantaged patients and their referring care providers that a living donor transplant is still very possibly the best option for an early transplant with an ideal outcome. Likewise, it is conceivable that candidates who have accumulated much dialysis time prior to listing represent a group with overall higher comorbidity [4, 14•] and risk for complication posttransplant and would similarly benefit greatly from a living donor transplant (Table 3).

Regarding the concern that back-dating wait list time to dialysis initiation may discourage patients from seeking early or pre-emptive listing, it should be noted the new system will still provide some opportunities for patients to receive the benefits of transplant without long wait times (Table 3). Candidates with zero HLA mismatched offers will continue to be prioritized above those with mismatched antigens, and highly sensitized patients (CPRA $>98 \%$ ) will also be prioritized. Also, the correlation between increased dialysis time and diminished transplant outcome has been well demonstrated [18]. Therefore, transplant professionals should continue to encourage care providers in the community to make timely referral and motivate patients to seek early listing for transplant.

Analyses of United States Renal Data System (USRDS) and UNOS data suggest underutilization of the voluntary national kidney allocation variance implemented in 2001 to permit OPO's to preferentially allocate A2 and A2B kidneys to B candidates to address the significantly longer median wait time for blood group B patients [12•, 13]. This is despite numerous reports of equivalent clinical outcomes for compatible vs. incompatible transplants using A2 kidneys and decreased wait times for B recipients of A2 kidneys [11, 19, 20]. An analysis of UNOS data indicates the proportion of A2 kidneys used for incompatible (A2 to B or O) transplants between 1997 and 2007 actually slightly decreased over time from 14.8 to $14.6 \%$ with regional disparity in frequency of A2 kidneys being used for incompatible transplants ranging from 6.7 to $46.2 \%$. While it is reported that approximately $20 \%$ of A donors are subtype A2, only slightly more than $3 \%$ were 
identified as A2 during the study period [12•]. A review of USRDS data on renal transplants performed between 1995 and 2006 found that less than half $(41.7 \%)$ of A donors had a subtype recorded [13]. These findings indicate that subtyping of all blood group A donors, an UNOS recommendation, is not routinely being performed. In order to optimize transplant opportunities under the new policy for disadvantaged blood group B candidates, OPO's should work with donor hospitals to make A subtyping universally available. Educational opportunities should be made available for blood group B candidates, who often represent minorities with long wait times, and their care providers to learn about potential benefits of receiving an $\mathrm{A} 2$ or A2B donor kidney. Transplant centers will need to coordinate with their blood banking experts to determine methodology, acceptable thresholds, and logistical considerations pertinent to checking anti-A titers and develop protocols and policies for eligibility for blood group B candidates to receive A2 and A2B kidneys [12•, 13, 20].

Successful transplantation of high CPRA patients in the new allocation era will be dependent upon effective use of recent advances in the field of histocompatibility. Widespread application of solid-phase technology in recent years has allowed precise identification of HLA specificities in sensitized patients. An analysis of OPTN crossmatch data for organ offers between 2001 and 2010 reported that following implementation of CPRA and widespread application of the virtual crossmatch, there was a nearly tenfold drop nationwide in the number of positive crossmatches reported as a reason for organ refusal. Concurrently, the percentage of kidneys transplanted in broadly sensitized patients (CPRA $>80 \%$ ) more than doubled, going from $7 \%$ to nearly $16 \%$. These trends have positive implications regarding cold ischemia time, resource conservation, cost savings, and organ utilization important for the next phase of kidney allocation prioritizing sensitized patients and employing broader sharing of kidneys for candidates with CPRA $>98 \%$ [10 $]$.

The lessons learned from the growing experience with transplanting highly sensitized patients through kidney paired donation (KPD) should be in large part translatable to high CPRA patients in the new allocation system. Approximately 2/3 of patients wait listed with the National Kidney Registry (NKR), a large multicenter KPD consortium, have CPRA $>80 \%$, and almost a third of more than 300 transplants facilitated by the NKR between March 2011 and December 2012 involved these highly sensitized patients. In reviewing their virtual crossmatch data for this time interval, it was found that $91 \%$ of virtual crossmatches accurately predicted a subsequent acceptable cell-based donor crossmatch. Nine percent of virtual crossmatches failed in that the donor was refused on the basis of an unacceptable cell-based crossmatch. In reviewing the reasons for failed virtual crossmatches, nearly half were attributed to equivocal virtual crossmatches resulting from DSA near the threshold for an unacceptable crossmatch or the cumulative effects of multiple weak DSAs that made prediction of the cell-based crossmatch unreliable. Twenty-one percent of failed virtual crossmatches were caused by changes in the recipient's HLA antibodies. Limitations in donor typing accounted for approximately $7 \%$ of virtual crossmatch failures and transcription errors were blamed for $6 \%$ of failures. The NKR experience reinforces important concepts for accurate virtual crossmatching. One is that comprehensive donor HLA typing (including HLA-DP and -DQA1 typing) is essential as sensitized patients have antibodies directed against the entire spectrum of HLA loci. It was felt that failures due to changes in the recipient's HLA antibody profile could be at least partially remedied by more frequent antibody testing. A consensus recommendation was for at least quarterly testing, with consideration for more frequent testing for highly sensitized patients or following sensitizing events. Unsuccessful virtual crossmatches as a result of errors in data entry have led to initiatives for laboratories to audit histocompatibility data [21•].

A positive cell-based crossmatch despite a negative virtual crossmatch may be explained by other reasons in addition to those discussed above. There may be failure to identify all anti-donor specificities which are clinically relevant due to limited solid phase testing. Alternatively, there may be nonHLA or autoantibodies present which may not necessarily be a contraindication for transplant [22]. Conversely, a negative cell-based result despite a positive virtual crossmatch may also be seen. This may, in some cases, be explained by presence of a weak DSA detected by highly sensitive solid phase testing. Transplant in this scenario may potentially expose the recipient to increased risk of long-term complications associated with DSA, and the decision to proceed or not needs to involve careful consideration of this risk, likelihood of other offers, and possible modification of the immunosuppressive protocol [22]. Other cases may be explained by diverse reasons including the antibody being directed to a different allele of the antigen group to which the donor HLA allele belongs but not to the allele expressed by the donor, presence of different alpha-beta chain combinations in the donor distinct from reagents used in the assay [22], or epitopes on denatured antigens which have become distorted in the process of being bound to a solid matrix [23•]. In these cases, despite the positive virtual crossmatch, it may be safe to proceed with transplant from the immunologic perspective $[22,23 \cdot]$. Suffice it to say that it will not be surprising for transplant programs to encounter a growing number of equivocal or discordant results regarding donor and recipient compatibility in the new era of allocation with prioritization of sensitized patients, and the insight of a dedicated histocompatibility expert to interpret these results and provide guidance as part of the transplant team will be invaluable and indispensable. 


\section{Access to Transplant for the Elderly}

Throughout the process of allocation policy development, there has been much discussion regarding the potential for diminished access to transplant for older candidates overall and in particular to higher quality organs in the interest of improving utility metrics (Table 3). The KTC Interim Report to the Board of Directors from January 2013 noted that previously considered proposals including life years from transplant (LYFT), age matching, national sharing, and combinations of these three approaches would have returned substantially more life years gained than the accepted proposal. In fact, the most aggressive proposal (national sharing + LYFT) would be projected to return approximately four times the life years achieved with the accepted proposal. However, this utility-driven approach would have allocated only $10 \%$ of transplants to candidates over the age of 50 . The accepted amendments provide $52 \%$ of transplants to candidates over the age of 50 . As patients over 50 make up $63 \%$ of the current waiting list, the KTC considered this a reasonable compromise between utility and equity. Although age is a major driver in determining EPTS, it is still substantially influenced by other factors. Data analysis presented concurrently by the KTC found that over $10 \%$ of candidates between ages 46 and 55 are estimated to be in the top $20 \%$ of EPTS. Also, the final amended allocation policy excludes pediatric candidates from EPTS calculation, thereby allowing approximately 700 more adults to be included in the EPTS top $20 \%$. Regarding organ quality, it may be argued that many kidneys from donors with KDPI scores outside the top $20 \%$ are still excellent kidneys. Good function may also be achieved from kidneys with higher than average KDPI scores.

More than half of recovered kidneys with KDPI $>85 \%$ are discarded under current practice. Broader sharing under the new system will hopefully increase utilization rates by offering these organs more efficiently with less ischemic time to centers more likely to use them. It is encouraging that analysis of early data presented at the August 2013 KTC meeting indicate that discard rates for the highest risk kidneys (KDPI $>96 \%$ ) have not increased since the time KDPI has been calculated and displayed for organ offers. There had been concerns that use of the continuous quality scale of donor quality in contrast to the previous dichotomous SCD/ECD system would discourage utilization of what would be labeled as the highest risk organs at the end of the scale. Approaches to improve utilization and outcomes of kidneys at higher risk of graft failure have been extensively reviewed in the literature including machine perfusion $[24,25]$ and dual kidney transplants [26-28] as well as appropriate listing of patients for these organs [29-33]. Extensive discussion of these topics is outside the realm of this paper but incorporation of these practices should be considered by OPO's and transplant programs as they strive to improve utilization and results achieved with these higher risk organs with implementation of the new policy.

Lastly, the questions of decreased access to deceased donor kidneys for older patients serve to highlight the benefits of living donor transplants in this population. Transplant with a living donor kidney may ameliorate the negative impact of comorbidity common in the elderly [34]. Older living donors may be particularly relevant for these candidates; data in this scenario support that clinical outcomes are favorable for both donor and recipient $[35,36]$.

\section{Conclusions}

To facilitate desired changes under the new kidney allocation system, it will be necessary for the transplant community to be aware of the goals and ramifications of the amended policy and actively engage patients as well as their care providers. In the best case scenario, "better" or more efficient allocation may result in more kidneys available for transplant. However, the demand will likely always far surpass the supply of transplantable deceased donor organs. Redistribution of a limited resource to enhance overall utility and improve equity for disadvantaged groups may be perceived as relatively diminished access for other groups. Similarly, re-stratification of the wait list may give rise to perceptions that potentially lead to unintended and undesirable consequences. With the implementation of the new policy, it will be important for transplant professionals to emphasize that the same benefits of living donor transplantation will still hold true for all patient subgroups as will the need for living donation. Early and preemptive referral and listing for transplant should continue to be encouraged as potential opportunities for early transplant exist through prioritization of zero mismatch offers and sensitized patients. The encouraging and increasing experience reported in the literature with the virtual crossmatch, KPD, and blood group A2 to B variance outline practical concepts and protocols which may be adapted and applied to the new allocation system to increase access for patients who are disadvantaged due to being highly sensitized or have blood group B.

The application of two granular metrics in the new allocation system, the KDPI and EPTS, as well as the sliding scale for points given to sensitized patients with CPRA $>20 \%$ provide more plasticity than present in the old system. The new system by nature of its flexibility should be amenable to modification based on outcomes without the need to completely overhaul the policy. Efforts will be soon underway to collect and analyze data under new allocation rules to determine if desired gains in utility as well as equity for previously disadvantaged subgroups are achieved. 
Compliance with Ethics Guidelines

Conflict of Interest Steven C. Kim, MD; Thomas C. Pearson, MD, DPhil; and Paul L. Tso, MD declare that they have no conflict of interest.

Human and Animal Rights and Informed Consent This article does not contain any studies with human or animal subjects performed by any of the authors.

\section{References}

Papers of particular interest, published recently, have been highlighted as:

- Of importance

-• Of major importance

1.• Israni AK, Salkowski N, Gustafson S, Snyder JJ, Friedewald JJ, Formica RN, et al. New national allocation policy for deceased donor kidneys in the United States and possible effect on patient outcomes. J Am Soc Nephrol JASN. 2014. This reference highlights the key changes in the new allocation guidelines approved by the OPTN. It also provides a simulated projection of how these changes will increase the projected median allograft years, improve overall post-transplant survival, as well as provide more access for highly sensitized candidates.

2.• Friedewald JJ, Samana CJ, Kasiske BL, Israni AK, Stewart D, Cherikh W, et al. The kidney allocation system. Surg Clin North Am. 2013;93(6):1395-406. This reference discusses limitations of current policy and changes being implemented in the new system. It highlights the compromises between utility and equity necessary in making new policy.

3. Rao PS, Schaubel DE, Guidinger MK, Andreoni KA, Wolfe RA, Merion RM, et al. A comprehensive risk quantification score for deceased donor kidneys: the kidney donor risk index. Transplantation. 2009;88(2):231-6.

4. Schold JD, Sehgal AR, Srinivas TR, Poggio ED, Navaneethan SD, Kaplan B. Marked variation of the association of ESRD duration before and after wait listing on kidney transplant outcomes. Am J Transplant Off J Am Soc Transplant Am Soc Transplant Surg. 2010;10(9):2008-16.

5. Wolfe RA, Ashby VB, Milford EL, Bloembergen WE, Agodoa LY, Held PJ, et al. Differences in access to cadaveric renal transplantation in the United States. Am J Kidney Dis Off J Natl Kidney Found. 2000;36(5):1025-33.

6. Young CJ, Gaston RS. African Americans and renal transplantation: disproportionate need, limited access, and impaired outcomes. Am J Med Sci. 2002;323(2):94-9.

7. Alexander GC, Sehgal AR. Barriers to cadaveric renal transplantation among blacks, women, and the poor. JAMA J Am Med Assoc. 1998;280(13):1148-52.

8. Kasiske BL, London W, Ellison MD. Race and socioeconomic factors influencing early placement on the kidney transplant waiting list. J Am Soc Nephrol JASN. 1998;9(11):2142-7.

9. Cecka JM. Calculated PRA (CPRA): the new measure of sensitization for transplant candidates. Am J Transplant Off J Am Soc Transplant Am Soc Transplant Surg. 2010;10(1):26-9.

10. Cecka JM, Kucheryavaya AY, Reinsmoen NL, Leffell MS. Calculated PRA: initial results show benefits for sensitized patients and a reduction in positive crossmatches. Am $\mathrm{J}$ Transplant Off $\mathrm{J}$ Am Soc Transplant Am Soc Transplant Surg. 2011;11(4):719-24
This article reports on promising early results with implementation of CPRA and virtual crossmatch.

11. Nelson PW, Shield 3rd CF, Muruve NA, Murillo D, Warady BA, Aeder MI, et al. Increased access to transplantation for blood group $\mathrm{B}$ cadaveric waiting list candidates by using A2 kidneys: time for a new national system? Am J Transplant Off J Am Soc Transplant Am Soc Transplant Surg. 2002;2(1):94-9.

12. Redfield RR, Parsons RF, Rodriguez E, Mustafa M, Cassuto J, Vivek K, et al. Underutilization of A2 ABO incompatible kidney transplantation. Clin Transpl. 2012;26(3):489-94. This article is an analysis of the utilization of the $A 2$ to $B$ variance based on UNOS data between 1997 and 2007.

13. Hurst FP, Sajjad I, Elster EA, Falta EM, Patel P, Abbott KC, et al. Transplantation of $\mathrm{A} 2$ kidneys into $\mathrm{B}$ and $\mathrm{O}$ recipients leads to reduction in waiting time: USRDS experience. Transplantation. 2010;89(11):1396-402.

14. Schold JD, Reese PP. Simulating the new kidney allocation policy in the United States: modest gains and many unknowns. J Am Soc Nephrol JASN. 2014. This editorial discusses reference \#1 pointing out potential shortcomings of the Kidney Pancreas Simulation Allocation Model (KPSAM).

15. Greenstein S, Siegal B. Compliance and noncompliance in patients with a functioning renal transplant: a multicenter study. Transplantation. 1998;66(12):1718-26.

16. Heaphy EL, Goldfarb DA, Poggio ED, Buccini LD, Flechner SM, Schold JD. The impact of deceased donor kidney risk significantly varies by recipient characteristics. Am J Transplant Off J Am Soc Transplant Am Soc Transplant Surg. 2013;13(4):1001-11.

17. Tullius SG, Tran H, Guleria I, Malek SK, Tilney NL, Milford E. The combination of donor and recipient age is critical in determining host immunoresponsiveness and renal transplant outcome. Ann Surg. 2010;252(4):662-74.

18. Meier-Kriesche HU, Kaplan B. Waiting time on dialysis as the strongest modifiable risk factor for renal transplant outcomes: a paired donor kidney analysis. Transplantation. 2002;74(10):1377-81.

19. Bryan CF, Winklhofer FT, Murillo D, Ross G, Nelson PW, Shield 3rd CF, et al. Improving access to kidney transplantation without decreasing graft survival: long-term outcomes of blood group A2/ A2B deceased donor kidneys in B recipients. Transplantation. 2005;80(1):75-80.

20. Nelson PW, Bryan CF. When will real benefits for minority patients be realized with A2->B transplants? Transplantation. 2010;89(11): 1310-1.

21. Baxter-Lowe LA, Cecka M, Kamoun M, Sinacore J, Melcher ML. Center-defined unacceptable HLA antigens facilitate transplants for sensitized patients in a multi-center kidney exchange program. Am J Transplant Off J Am Soc Transplant Am Soc Transplant Surg. 2014;14(7):1592-8. This article analyzes the use of virtual crossmatch in highly sensitized patients- the reasons and potential solutions for instances of failure.

22. Tambur AR, Ramon DS, Kaufman DB, Friedewald J, Luo X, Ho B, et al. Perception versus reality? Virtual crossmatch - how to overcome some of the technical and logistic limitations. Am J Transplant Off J Am Soc Transplant Am Soc Transplant Surg. 2009;9(8):1886-93.

23. Tait BD, Susal C, Gebel HM, Nickerson PW, Zachary AA, Claas $\mathrm{FH}$, et al. Consensus guidelines on the testing and clinical management issues associated with HLA and non-HLA antibodies in transplantation. Transplantation. 2013;95(1):19-47. This is a comprehensive consensus paper on current histocompatibility topics relevant to clinicians.

24. Schold JD, Kaplan B, Howard RJ, Reed AI, Foley DP, MeierKriesche HU. Are we frozen in time? Analysis of the utilization and efficacy of pulsatile perfusion in renal transplantation. Am J Transplant Off J Am Soc Transplant Am Soc Transplant Surg. 2005;5(7):1681-8. 
25. Sung RS, Christensen LL, Leichtman AB, Greenstein SM, Distant DA, Wynn JJ, et al. Determinants of discard of expanded criteria donor kidneys: impact of biopsy and machine perfusion. Am J Transplant Off J Am Soc Transplant Am Soc Transplant Surg. 2008;8(4):783-92.

26. Ekser B, Furian L, Broggiato A, Silvestre C, Pierobon ES, Baldan $\mathrm{N}$, et al. Technical aspects of unilateral dual kidney transplantation from expanded criteria donors: experience of 100 patients. Am J Transplant Off J Am Soc Transplant Am Soc Transplant Surg. 2010;10(9):2000-7.

27. Gill J, Cho YW, Danovitch GM, Wilkinson A, Lipshutz G, Pham PT, et al. Outcomes of dual adult kidney transplants in the United States: an analysis of the OPTN/UNOS database. Transplantation. 2008;85(1):62-8.

28. Remuzzi G, Cravedi P, Perna A, Dimitrov BD, Turturro M, Locatelli $\mathrm{G}$, et al. Long-term outcome of renal transplantation from older donors. N Engl J Med. 2006;354(4):343-52.

29. Grams ME, Womer KL, Ugarte RM, Desai NM, Montgomery RA, Segev DL. Listing for expanded criteria donor kidneys in older adults and those with predicted benefit. Am J Transplant Off J Am Soc Transplant Am Soc Transplant Surg. 2010;10(4):802-9.

30. Cecka JM, Cohen B, Rosendale J, Smith M. Could more effective use of kidneys recovered from older deceased donors result in more kidney transplants for older patients? Transplantation. 2006;81(7): 966-70.
31. Merion RM, Ashby VB, Wolfe RA, Distant DA, Hulbert-Shearon TE, Metzger RA, et al. Deceased-donor characteristics and the survival benefit of kidney transplantation. JAMA J Am Med Assoc. 2005;294(21):2726-33.

32. Frei U, Noeldeke J, Machold-Fabrizii V, Arbogast H, Margreiter R, Fricke L, et al. Prospective age-matching in elderly kidney transplant recipients - a 5-year analysis of the Eurotransplant Senior Program. Am J Transplant Off J Am Soc Transplant Am Soc Transplant Surg. 2008;8(1):50-7.

33. Carter JT, Chan S, Roberts JP, Feng S. Expanded criteria donor kidney allocation: marked decrease in cold ischemia and delayed graft function at a single center. Am J Transplant Off J Am Soc Transplant Am Soc Transplant Surg. 2005;5(11):2745-53.

34. Wu C, Shapiro R, Tan H, Basu A, Smetanka C, Morgan C, et al. Kidney transplantation in elderly people: the influence of recipient comorbidity and living kidney donors. J Am Geriatr Soc. 2008;56(2):231-8.

35. Segev DL, Muzaale AD, Caffo BS, Mehta SH, Singer AL, Taranto SE, et al. Perioperative mortality and long-term survival following live kidney donation. JAMA J Am Med Assoc. 2010;303(10):95966.

36. Gill J, Bunnapradist S, Danovitch GM, Gjertson D, Gill JS, Cecka M. Outcomes of kidney transplantation from older living donors to older recipients. Am J Kidney Dis Off J Natl Kidney Found. 2008;52(3):541-52. 